\title{
Dynamic colloidal membranes
}

\author{
Stephen S. L. Peppin
}

\begin{abstract}
A model of the formation and mechanics of a colloidal membrane is obtained within the framework of the hydrodynamic theory of cross diffusion. Coupled equations describing advection, diffusion and cross-diffusion of spherical tracer particles within a colloidal suspension are solved numerically. A dynamic membrane is formed by advecting the colloidal particles toward a porous support; as the particles build up against the support they form a concentrated boundary layer; at sufficiently high concentrations this layer behaves like a filtration membrane. A tracer pulse is advected toward the steady-state colloidal membrane; the tracer particles are filtered by the colloidal particles via excluded volume and hydrodynamic interactions, quantified by the colloidal reflection coefficient. The filtered tracer particles form a secondary concentrated boundary layer within the membrane. Decreasing the size of the tracer particles allows them to penetrate further into the colloidal membrane, and finally to pass through below a critical size threshold.
\end{abstract}

\section{Introduction}

Ultrafiltration is a membrane separation process used extensively in industry to purify water, process pharmaceuticals and concentrate milk and juices [1]. Ultrafiltration also occurs in many physiological and natural settings [2,3]. In ultrafiltration systems a colloidal suspension is advected toward a membrane or porous support that is impermeable to the colloidal particles, but through which the pore fluid and molecular impurities can pass (figure 1). During the filtration process the colloidal particles build up adjacent to the membrane forming a concentration polarization layer. The large colloid osmotic pressure in this layer can lead to detrimental effects such as membrane fouling and flux decline [4]. On the other hand if the colloidal particles exhibit membrane properties, the concentration polarization layer itself becomes a secondary dynamic filtration membrane that can be used to remove tracer particles from the pore fluid [5-7]. Recent experimental studies demonstrate the excellent potential of dynamic colloidal membranes for water purification applications [5,8]. The membranes form naturally, are relatively inexpensive to produce and can be easily removed and regenerated, mitigating the effects of membrane fouling. A significant advantage of such systems is that expensive ultrafiltration membranes are not required, since the secondary dynamic membrane performs the filtration.

In this work a model of a colloidal membrane is developed within the context of the hydrodynamic theory of diffusion and cross-diffusion in suspensions $[9,10]$. In Section 2 the diffusive fluxes and mass conservation equations are introduced and put in dimensionless form. In Section 3 the equations are solved for the case of a one-dimensional filtration system with two different sets of boundary conditions, corresponding to self-forming and pre-deposited dynamic membranes. Section 4 contains a discussion of the results and conclusions.

\section{Mass conservation in binary suspensions}

Conservation of mass in two-component suspensions can be written as

$$
\frac{\partial n_{1}}{\partial t}+\mathbf{u} \cdot \boldsymbol{\nabla} n_{1}=-\nabla \cdot \mathbf{J}_{1} \quad \text { and } \quad \frac{\partial n_{2}}{\partial t}+\mathbf{u} \cdot \nabla n_{2}=-\boldsymbol{\nabla} \cdot \mathbf{J}_{2},
$$

For God so loved the world, that He gave His only begotten Son, that whosoever believeth in Him should not perish, but have everlasting life. For God sent not His Son into the world to condemn the world; but that the world through Him might be saved. John 3:16-17 
(a)

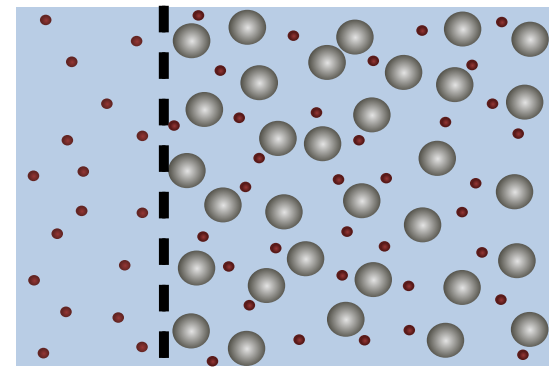

(b)

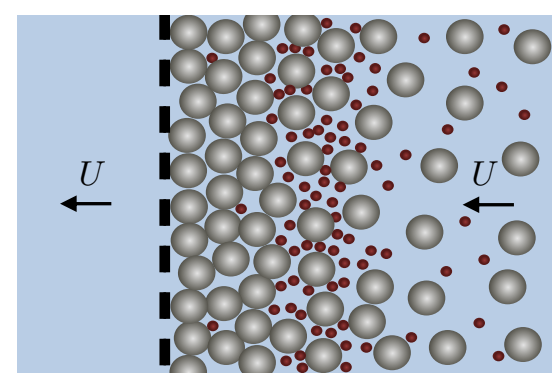

Figure 1: Schematic of a self-forming dynamic colloidal membrane [5,7]. (a) A suspension of initial volume fraction $\phi_{1}^{0}$ of colloidal particles (grey) and $\phi_{2}^{0}$ of tracer particles (red) is placed adjacent to a membrane or porous support. The membrane is impermeable to the colloidal particles, but permeable to the tracer particles. (b) A flow $\mathbf{u}=-U \hat{\mathbf{i}}$ is imposed, consolidating the colloidal particles against the support; the colloidal particles form a dynamic membrane that filters the tracer particles from the pore fluid.

where $n_{i}$ is the concentration (number of particles per unit volume) of component $i$, $\mathbf{u}$ is the volume-average velocity of the mixture and $\mathbf{J}_{i}=n_{i}\left(\mathbf{u}_{i}-\mathbf{u}\right)$ is the diffusive flux of $i$ relative to $\mathbf{u}$. In systems experiencing strong cross-diffusion effects the fluxes take the form

$$
\mathbf{J}_{1}=-D_{11} \boldsymbol{\nabla} n_{1}-D_{12} \boldsymbol{\nabla} n_{2} \quad \text { and } \quad \mathbf{J}_{2}=-D_{21} \boldsymbol{\nabla} n_{1}-D_{22} \boldsymbol{\nabla} n_{2},
$$

where $D_{11}$ and $D_{22}$ are the main Fickian diffusion coefficients of the colloidal particles and tracer particles, and $D_{12}$ and $D_{21}$ are cross-diffusion coefficients. Expressions for the $D_{i j}$ as functions of particle concentration have been obtained previously for hard-sphere suspensions [9-11] and for polymer solutions [12]. Here the hard-sphere expressions are used, as summarized in the Appendix.

\subsection{Governing equations}

Figure $1 a$ shows a schematic of the system to be studied. At $x=0$ is a porous support or mesh that is permeable to the fluid and tracer particles, but impermeable to the colloidal particles. Adjacent to the support is a colloidal suspension of initial concentration $n_{1}^{0}$ containing tracer particles at $n_{2}^{0}$. At $t=0$ a volume flow $\mathbf{u}=-U \hat{\mathbf{i}}$ is imposed and the colloidal particles are advected toward the porous support, forming a concentration polarization layer (figure $1 b$ ). The boundary conditions at $x=0$ are that $\mathbf{u}_{1}=0$ and $\mathbf{u}_{2}=-U \hat{\mathbf{i}}$. In terms of the fluxes $\mathbf{J}_{i}$ this gives

$$
\mathbf{J}_{1}=n_{1} U \hat{\mathbf{i}} \quad \text { and } \quad \mathbf{J}_{2}=0 \quad(x=0) .
$$

For the one-dimensional system in figure 1, conservation of mass equations (1) take the form

$$
\frac{\partial n_{1}}{\partial t}-U \frac{\partial n_{1}}{\partial x}=-\frac{\partial J_{1}}{\partial x} \quad \text { and } \quad \frac{\partial n_{2}}{\partial t}-U \frac{\partial n_{2}}{\partial x}=-\frac{\partial J_{2}}{\partial x} \quad(x>0) \text {, }
$$

and the fluxes (2) become

$$
J_{1}=n_{1}\left(u_{1}+U\right)=-D_{11} \frac{\partial n_{1}}{\partial x}-D_{12} \frac{\partial n_{2}}{\partial x}
$$


and

$$
J_{2}=n_{2}\left(u_{2}+U\right)=-D_{21} \frac{\partial n_{1}}{\partial x}-D_{22} \frac{\partial n_{2}}{\partial x} .
$$

It will be convenient numerically to make the equations dimensionless. This can be accomplished by introducing the dimensionless lengths $\hat{x}=x / L$ and times $\hat{t}=t U / L$; dimensionless velocities $\hat{u}_{i}=u_{i} / U$; volume fractions $\phi_{i}=n_{i} v_{i}$, where $v_{i}=\frac{4}{3} \pi R_{i}^{3}$ is the particle volume and $R_{i}$ the particle radius; dimensionless fluxes $\hat{J}_{i}=v_{i} J_{i} / U$; and dimensionless diffusivities $\hat{D}_{i j}=D_{i j} / D_{i}^{0}$, where $D_{i}^{0}=k_{B} T /\left(6 \pi \eta_{0} R_{i}\right)$ is the Stokes-Einstein diffusivity of a particle and $\eta_{0}$ is the viscosity of the pore fluid. In terms of these quantities equations (4)-(6) take the dimensionless form

$$
\begin{array}{ll}
\frac{\partial \phi_{1}}{\partial \hat{t}}=\frac{\partial \phi_{1}}{\partial \hat{x}}-\frac{\partial \hat{J}_{1}}{\partial \hat{x}} & (\hat{x}>0), \\
\frac{\partial \phi_{2}}{\partial \hat{t}}=\frac{\partial \phi_{2}}{\partial \hat{x}}-\frac{\partial \hat{J}_{2}}{\partial \hat{x}} \quad(\hat{x}>0),
\end{array}
$$

where

$$
\hat{J}_{1}=-\frac{1}{P e}\left(\hat{D}_{11} \frac{\partial \phi_{1}}{\partial \hat{x}}+\lambda^{-3} \hat{D}_{12} \frac{\partial \phi_{2}}{\partial \hat{x}}\right)
$$

and

$$
\hat{J}_{2}=-\frac{1}{\lambda P e}\left(\lambda^{3} \hat{D}_{21} \frac{\partial \phi_{1}}{\partial \hat{x}}+\hat{D}_{22} \frac{\partial \phi_{2}}{\partial \hat{x}}\right)
$$

In equations (9) and (10) $P e=U L / D_{1}^{0}$ is the Peclet number and $\lambda=R_{2} / R_{1}=D_{1}^{0} / D_{2}^{0}$ is the particle size ratio. The boundary conditions (3) become

$$
\hat{J}_{1}=\phi_{1} \quad \text { and } \quad \hat{J}_{2}=0 \quad(\hat{x}=0) .
$$

The far-field boundary conditions for the system in figure 1 are

$$
\phi_{1} \rightarrow \phi_{1}^{0} \quad \text { and } \quad \phi_{2} \rightarrow \phi_{2}^{0} \quad(\hat{x} \rightarrow \infty),
$$

where $\phi_{i}^{0}=n_{i}^{0} v_{i}$ is the initial volume fraction of component $i$ in the suspension.

\section{Results}

\subsection{Self-forming dynamic membrane}

Figure 2 shows the results of a simulation of a self-forming dynamic membrane, in which a suspension with initial concentrations $\phi_{1}^{0}=0.1$ and $\phi_{2}^{0}=0.001$, size ratio $\lambda=0.7$ and Peclet number $P e=20$, is advected toward the porous support. The equations have been solved using the MatLab partial differential equation solver pdepe (Matlab files included as Supplementary Material). At early times $(\hat{t}=0.05$ and $\hat{t}=0.1)$, the colloid volume fraction increases rapidly against the support at $\hat{x}=0$ (figure $2 \mathrm{a}$ ), but the tracer particle concentration is relatively unchanged from it's initial value $\phi_{2}^{0}$ (figure $2 \mathrm{~b}$ ). The colloidal suspension is still relatively porous at this stage and the tracer particles are able to pass through into the filtrate. However, as the colloid is further consolidated $(\hat{t}=0.15)$, the volume fraction at $\hat{x}=0$ approaches close packing and the tracer particles can no longer easily fit through the pore space. At longer times $(\hat{t} \gtrsim 0.2)$ the colloidal membrane almost completely blocks the tracer particles, and a large tracer polarization layer forms within the membrane. The inset to figure $2 \mathrm{~b}$ shows the time evolution of the tracer concentration entering the filtrate at $\hat{x}=0$. Once the dynamic membrane has formed $(\hat{t} \gtrsim 0.1)$, the tracer concentration entering the filtrate drops rapidly, approaching zero at longer times. 

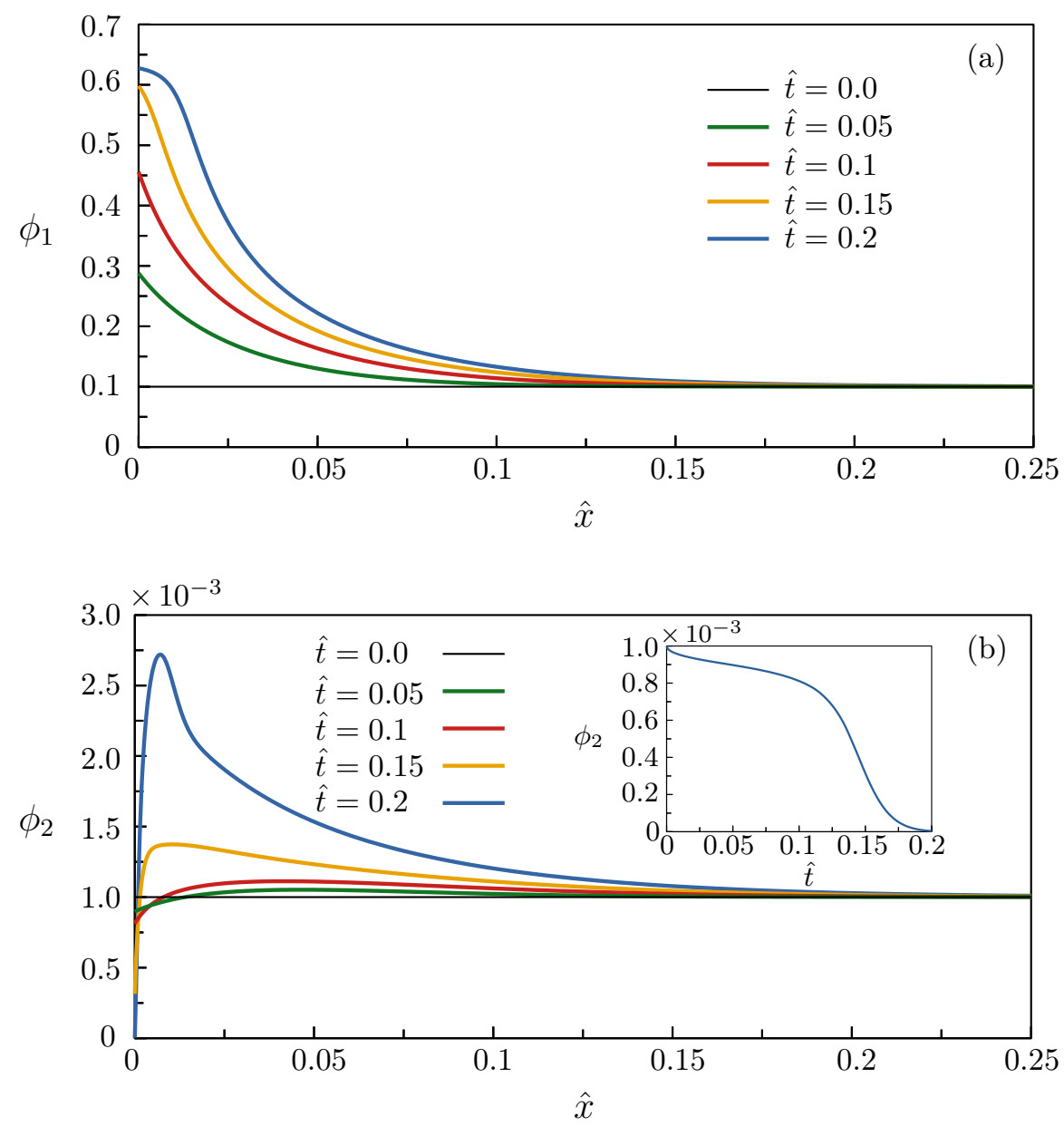

Figure 2: Simulation of a self-forming dynamic membrane, in which a binary suspension with $\lambda=0.7$ and initial concentrations $\phi_{1}^{0}=0.1$ and $\phi_{2}^{0}=0.001$ is advected toward a porous support at $\hat{x}=0$. The inset to (b) shows the tracer concentration at $\hat{x}=0$ as a function of time.

\subsection{Pre-deposited colloidal membrane}

In the next simulation, with results shown in figure 3, a layer of colloidal particles of initial concentration $\phi_{1}^{0}$ and width $L$ is first advected toward the porous support, in the absence of the tracer particles, in order to form a pre-deposited dynamic membrane [7]. In this case $\phi_{2}=0$ and equations (7)-(10) reduce to the classic ultrafiltration equations studied by Russel et al. [13, 14]:

$$
\frac{\partial \phi_{1}}{\partial \hat{t}}=\frac{\partial \phi_{1}}{\partial \hat{x}}+\frac{1}{P e} \frac{\partial}{\partial \hat{x}}\left(\hat{D}_{11} \frac{\partial \phi_{1}}{\partial \hat{x}}\right) \quad(\hat{x}>0),
$$

with boundary conditions

$$
P e \phi_{1}=-\hat{D}_{11} \frac{\partial \phi_{1}}{\partial \hat{x}} \quad(\hat{x}=0,1) .
$$

Figure 3 shows the solution at various times approaching steady state for the case $\phi_{0}=0.1$ and $P e=20$. Similarly to Davis and Russel [13], the concentration at $\hat{x}=0$ quickly approaches the close-packing value $\phi_{p}=0.64$, and a dynamic membrane with diffuse boundary layer forms; at $\hat{t} \gtrsim 10$ the system has reached steady state. The steady-state porosity $n=1-\phi_{1}$ of the dynamic membrane is lowest at $\hat{x}=0$ and increases significantly within the diffuse boundary layer. As will be seen in the next section, the varying porosity of the dynamic membrane allows a tracer pulse to penetrate some distance within the membrane before being blocked by the reduction in pore size. 


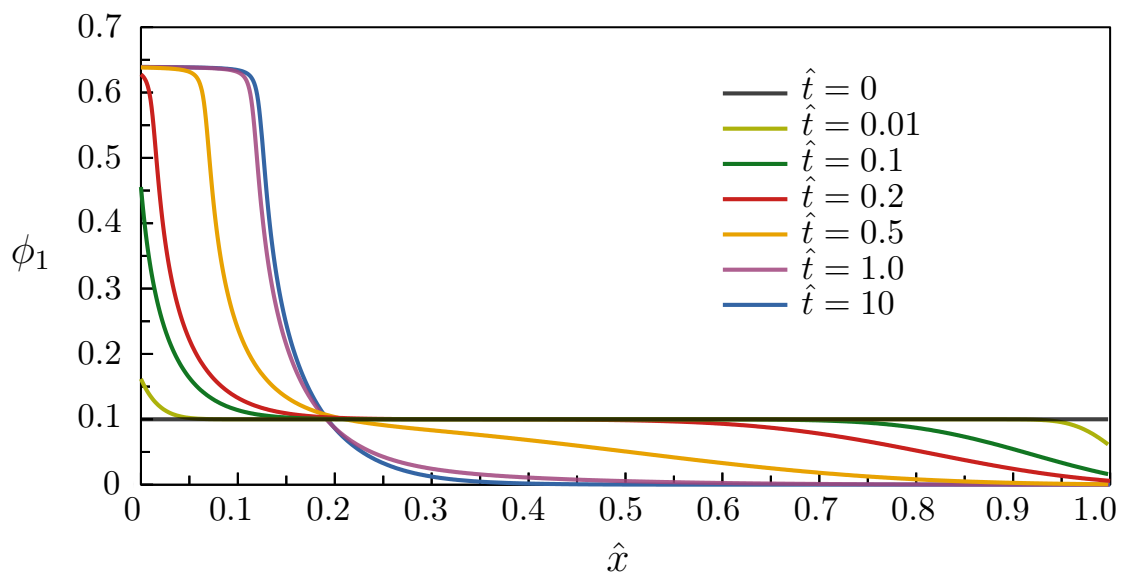

Figure 3: Simulation of the development of a pre-deposited colloidal membrane by advecting a layer of colloidal particles with initial volume fraction $\phi_{1}^{0}=0.1$ against a porous support at $\hat{x}=0$. At $\hat{t}=10$ the dynamic membrane has reached a steady-state configuration.

\subsubsection{Colloidal filtration of a Gaussian tracer pulse}

In figure 4 a Gaussian pulse of tracer particles of the form $\left.\phi_{2}=\phi_{2}^{0} \exp \left[-a\left(\hat{x}-\hat{x}_{0}\right)^{2}\right)\right]$ is introduced at $\hat{x}=\hat{x}_{0}$ and advected toward the steady-state dynamic membrane obtained in figure 3 , which is used as an initial condition for $\phi_{1}(\hat{x})$. Figure 4 a shows results for the case $\phi_{2}^{0}=0.001, a=2000$ and $\hat{x}_{0}=0.4$, with $P e=20$ and $\lambda=2$. At early times the tracer plume experiences traditional advection-diffusion effects, with the plume spreading diffusively as it moves towards the membrane. As the tracer particles enter the membrane, however, the restricted pore space begins to hinder their motion. Once the tracer particles reach a critical depth within the membrane they are unable to advect further, and they form a concentrated tracer boundary layer. Physically, the tracer particles are blocked by the membrane because the pore size of the membrane eventually becomes smaller than the tracer particle size, as discussed further in Section 4.

As a comparison and to illustrate the effects of cross-diffusion on the system behaviour, figure $4 \mathrm{~b}$ shows results under identical conditions, but with the cross-diffusion coefficients set to zero, $D_{12}=D_{21}=0$. In this case, the tracer particles are able to pass through the membrane. The tracer diffusivity $D_{22}$ still approaches zero at a critical depth within the dynamic membrane and the tracer particles no longer experience Brownian diffusion. This can be seen in the profiles for $\hat{t}>0.2$, where the width of the tracer plume remains fixed as it moves through the membrane. While diffusion ceases, the plume is still able to advect through the membrane as a travelling wave, and eventually passes through the system and into the filtrate $(\hat{t}=0.5)$.

Figure 5 shows the effect of changing the size of the tracer particles on the $\hat{t}=0.5$ profile in figure 4a. The solid curves show the tracer concentration profile at $\hat{t}=0.5$ for several values of the particle size ratio $\lambda=R_{2} / R_{1}$. As the size ratio decreases the tracer particles are able to reach further into the dynamic membrane, until for $\lambda \lesssim 0.4$ they are able to pass through into the filtrate.

\section{Discussion}

The membrane effects in figures 2-5 are caused by the cross-diffusion $D_{21}$ term in the tracer flux equation $(1 b)$. In the ultrafiltration system in figure $2, \nabla n_{1}<0$ while $\nabla n_{2}>0$ at $\hat{x}=0$, and the two terms on the right hand side of $(1 b)$ are of opposite sign. The main $D_{22}$ diffusion term acts to relax the tracer concentration gradient and move the particles through the membrane in the $-\hat{x}$ direction. The cross-diffusion term is driving them in the opposite direction, against their own concentration gradient (uphill diffusion) [11]. When the colloid volume fraction $\phi_{1}$ approaches close-packing, hindered diffusion causes the tracer Fickian diffusion coefficient $D_{22}$ to reduce to zero (figure $7 d$ ). The cross-diffusion coefficient $D_{21}$, however, becomes large in this limit (figure $7 c$ ) and uphill diffusion dominates, prohibiting the tracer particles from passing through the membrane. 

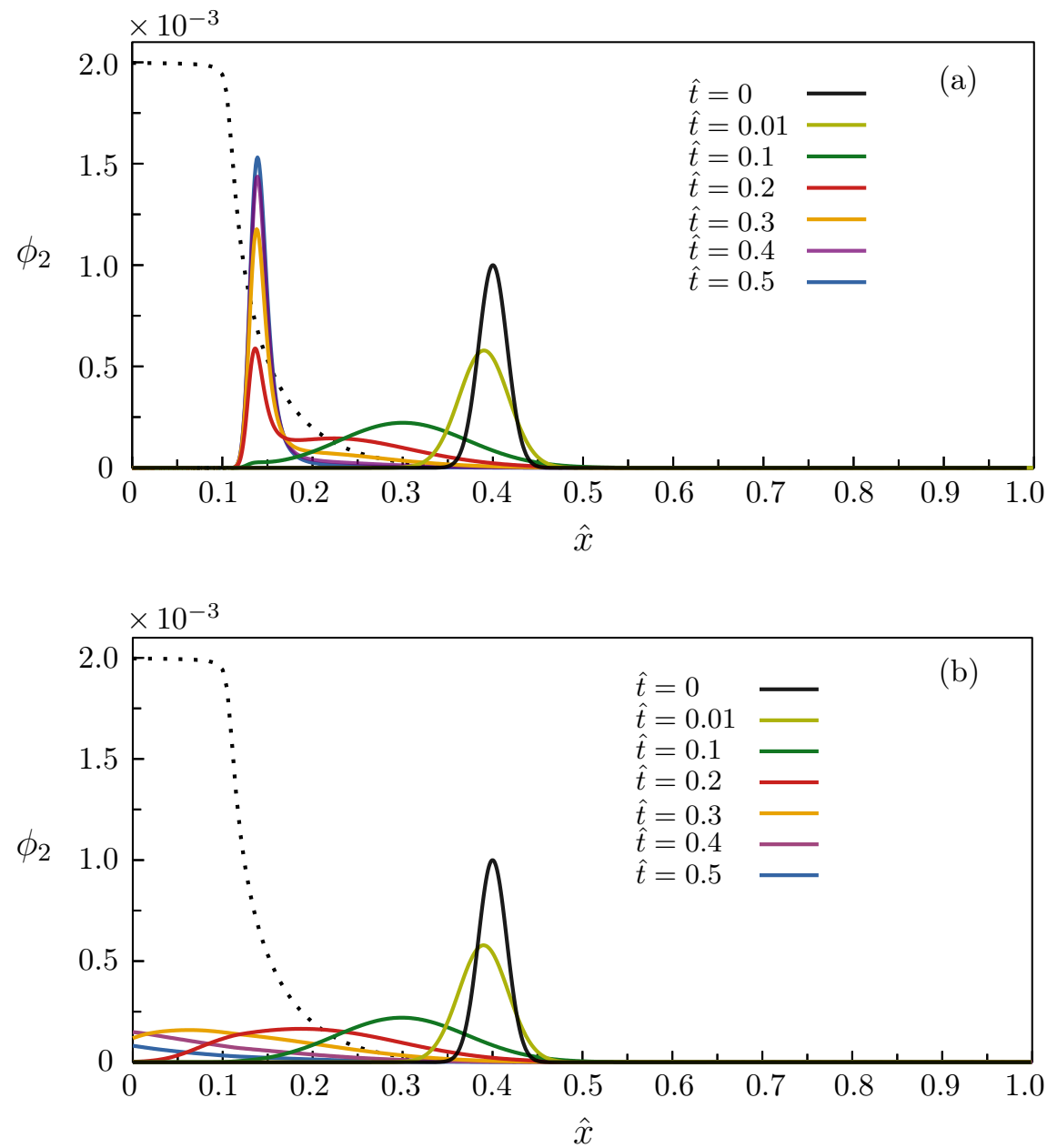

Figure 4: Simulation of the advection of a tracer pulse toward a dynamic colloidal membrane, for the case $P e=20$ and $\lambda=2$. In (a) cross-diffusion effects stop the tracer pulse from moving through the membrane. In (b) the cross-diffusion coefficients are set to zero and the tracer is able to pass through the system into the filtrate. The dotted curve shows the steady-state position of the dynamic membrane obtained in figure 3 .

The filtration mechanism of the colloidal membrane can be further illustrated by considering the advection of a single tracer particle within the steady-state membrane. In this case $u_{1}=0$, and for a single tracer particle $\partial \phi_{2} / \partial x=0$. The flux equations (5) and (6) become

$$
n_{1} U=-D_{11} \frac{\partial n_{1}}{\partial x}
$$

and

$$
n_{2}\left(u_{2}+U\right)=-D_{21} \frac{\partial n_{1}}{\partial x} .
$$

As the tracer moves into the membrane the pore size decreases and the tortuosity factor $\tau$ approaches 0 while the reflection coefficient $\sigma$ approaches 1 (see Appendix); the cross-diffusion factor $\ell=(\sigma+\alpha-1) / \alpha$ also approaches 1 in this limit, so that the tracer cross-diffusion coefficient (18c) becomes $D_{21}=D_{2}^{0} K \hat{\Pi}_{1}\left(\phi_{2} / \lambda^{2} \phi_{1}\right)=D_{11} n_{2} / n_{1}$. Substituting this into (16) and solving for the tracer velocity $u_{2}$ then gives

$$
u_{2}=-U-\frac{D_{21}}{n_{2}} \frac{\partial n_{1}}{\partial x}=-U+U \frac{n_{1} D_{21}}{n_{2} D_{11}}=0 .
$$

Therefore, the tracer particle is trapped and unable to move within the membrane when $\tau=0$ and $\sigma=1[10]$. 


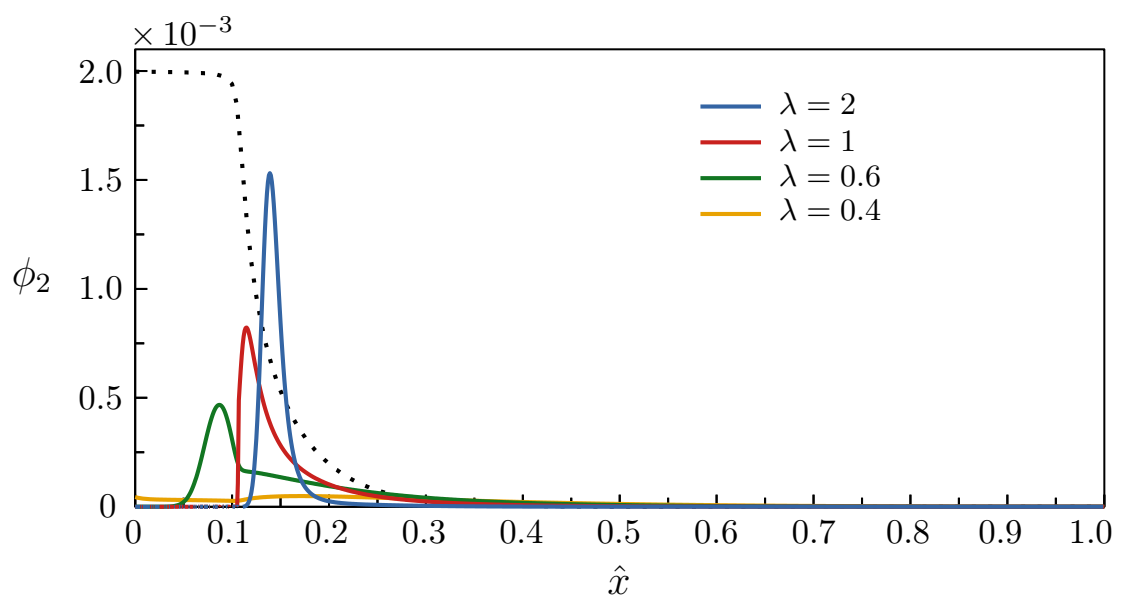

Figure 5: Illustration of the effect of changing the particle size ratio $\lambda=R_{2} / R_{1}$ on the tracer profiles within the dynamic membrane. The blue curve $(\lambda=2)$ shows the final profile obtained in figure $4 \mathrm{a}(\hat{t}=0.5)$. As $\lambda$ decreases (red, green and yellow curves) the tracer particles are able to reach further into the membrane; for $\lambda \lesssim 0.4$ the tracer particles are no longer completely blocked and can pass through into the filtrate.

Quantitatively, the model results depend on the expressions for the cross-diffusion coefficients summarized in the Appendix. Expressions for $D_{11}$ and $D_{22}$ have been compared with experiment for the hard-sphere system [10,11], and similar equations for all four $D_{i j}$ are in good agreement with experiments on salt cross diffusion in polyethylene glycol solutions [12]. Perhaps the most uncertain aspect of the model is the expression for the colloidal reflection coefficient $\sigma$. In this work an expression (22b) for $\sigma$ obtained previously [10] for hard-sphere suspensions has been used. An alternative expression for $\sigma$ relevant to porous media was also obtained in [10] and may be more appropriate for dynamic membranes as it ensures the tracer particles are blocked by the membrane whenever the particle size is equal to or greater than the physical pore size. However, either expression gives qualitatively similar results $\left(u_{2} \rightarrow 0\right.$ as $\tau \rightarrow 0$ and $\sigma \rightarrow 1$ ). Expressions for $\sigma$ that also depend on particle charge and shape in cylindrical pores have been obtained by Anderson and Quinn [15] and Bhalla and Deen [16], which have a qualitatively similar behaviour to the expression used here $(\sigma \rightarrow 0$ as the pore size increases while $\sigma \rightarrow 1$ as the pore size approaches the tracer particle size).

\section{Conclusions}

A model of a dynamic colloidal membrane has been developed within the framework of the hydrodynamic theory of cross diffusion. The model simulates two experimental configurations: a self-forming membrane in which the colloidal particles are part of the feed solution; and a pre-deposited membrane with subsequent filtration of a tracer plume. The filtration effect of the colloidal membrane is caused by strong cross-diffusion effects, which tend to push the tracer particles in a direction opposite to their own concentration gradient (uphill diffusion). The model accounts for the effects of hydrodynamic and excluded volume interactions between the colloidal membrane and tracer particles, and simulates the effect of varying the tracer particle size. For sufficiently small particles, the tracers can pass through the pores of the membrane; once the tracer radius approaches the pore size, however, the tracer advection is hindered, and the particles instead form a concentrated boundary layer within the membrane. The generic hard-sphere model developed here should provide a basic framework within which to study dynamic membrane effects in more complex systems. 


\section{Acknowledgements}

This work was made possible by the love and merciful kindness of Jesus, Lord of heaven and earth and Saviour of all who believe. Believe on the Lord Jesus Christ and thou shalt be saved, and thy house. Acts 16:31.

\section{A Diffusion coefficients}

For tracer diffusion in concentrated suspensions the four cross-diffusion coefficients can be written in the form

$$
\begin{gathered}
D_{11}=D_{1}^{0} K \hat{\Pi}_{1}, \quad D_{12}=D_{1}^{0} K \sigma / \alpha, \\
D_{21}=D_{2}^{0}\left(\hat{\ell} K \hat{\Pi}_{1}-\gamma \tau\right), \quad D_{22}=D_{2}^{0} \tau,
\end{gathered}
$$

where $D_{i}^{0}=k_{B} T /\left(6 \pi R_{i} \eta_{0}\right)$ is the Stokes-Einstein diffusivity of particle $i, k_{B}$ is Boltzmann's constant, $T$ is temperature, and $\eta_{0}$ is the viscosity of the suspending fluid [10]. In (18a) $K$ is the dimensionless permeability (viscous mobility) of the host-particle matrix and $\hat{\Pi}_{1}=\Pi_{1} /\left(k_{B} T\right)=$ $\mathrm{d}\left(\phi_{1} Z\right) / \mathrm{d} \phi_{1}$, where $\Pi_{1}=\left(\partial \Pi / \partial n_{1}\right)_{T}$ is the derivative of the host-matrix osmotic pressure $\Pi$ with respect to concentration; $\phi_{1}=n_{1} v_{1}$ is the host-particle volume fraction, $v_{1}=\frac{4}{3} \pi R_{1}^{3}$ is the volume of a host particle and $Z\left(\phi_{1}\right)$ is the host-matrix compressiblity factor. In $(18 b) \sigma$ is the reflection coefficient of the host matrix accounting for hindrance effects on tracer advection within the pore space, and $\alpha$ is the equilibrium partition coefficient of the tracer particles between the pore space and the bulk fluid. In $(18 c) \gamma$ is a preferential interaction coefficient accounting for excluded volume effects; and $\hat{\ell}=\phi_{2} \ell /\left(\lambda^{2} \phi_{1}\right)$, where $\ell=(\alpha+\sigma-1) / \alpha$ is the viscous cross-diffusion factor between the tracer and host particles, $\phi_{2}=n_{2} v_{2}$ is the tracer-particle volume fraction and $\lambda=R_{2} / R_{1}$ is the ratio of particle sizes. Finally, in $(18 d) \tau$ is the diffusive tortuosity factor accounting for the hindrance effect on tracer diffusion within the pore space.

For spherical particles approximate expressions for the friction and compressibility factors of the host-particle matrix are

$$
K=\left(1-\phi_{1}\right)^{6.55} \quad \text { and } \quad Z=\frac{1+a \phi_{1}}{1-\phi_{1} / \phi_{p}}
$$

where $a=4-1 / \phi_{p}$ and $\phi_{p}=0.64$ is the volume fraction at random close packing [11]. In the dilute limit $\phi_{1} \ll 1$ equations (19) reduce to the exact results $K=1-6.55 \phi_{1}$ and $Z=1+4 \phi_{1}[17,18]$. Given $Z, \hat{\Pi}_{1}$ can be obtained as

$$
\hat{\Pi}_{1}=\frac{\mathrm{d}\left(\phi_{1} Z\right)}{\mathrm{d} \phi_{1}}=\frac{1+2 a \phi_{1}-a \phi_{1}^{2} / \phi_{p}}{\left(1-\phi_{1} / \phi_{p}\right)^{2}} .
$$

Approximate expressions for the partition coefficient $\alpha$ and preferential interaction coefficient $\gamma$ in hard-sphere suspensions are

$$
\alpha=\left(1-\phi_{2} / \phi_{p}\right) \mathrm{e}^{-b_{12} \phi_{1}}+\phi_{2} / \phi_{p} \quad \text { and } \quad \gamma=-n_{2} b_{12} v_{1}=-\phi_{2} b_{12} / \lambda^{3},
$$

where $\lambda=R_{2} / R_{1}$ is the size ratio of the tracer and host particles, $b_{12}=(1+\lambda)^{3}$ is a thermodynamic coupling coefficient, $\phi_{2}=n_{2} v_{2}$ is the tracer volume fraction and $v_{2}=\frac{4}{3} \pi R_{2}^{3}$ is the tracer particle volume $[10,11]$.

Constitutive equations for the diffusive tortuosity factor $\tau$ and reflection coefficient $\sigma$ in hard sphere suspensions are

$$
\tau=\left\{\begin{array}{ll}
\left(1-\lambda_{d}\right)^{a_{\tau}}, & \text { if } 0 \leq \lambda_{d} \leq 1 \\
0 & \text { if } \lambda_{d}>1
\end{array} \quad \text { and } \quad \sigma= \begin{cases}1-\left(1-\lambda_{d}\right)^{a_{\sigma}}, & \text { if } 0 \leq \lambda_{d} \leq 1 \\
1 & \text { if } \lambda_{d}>1\end{cases}\right.
$$

where

$$
\lambda_{d}=\frac{3 \lambda \phi_{1}}{c_{d}\left(1-\phi_{1}\right)} \quad \text { and } \quad c_{d}=3 \frac{\phi_{g}}{\left(1-\phi_{g}\right)} \frac{\left(1+\lambda^{2}\right)}{(1+\lambda)}
$$



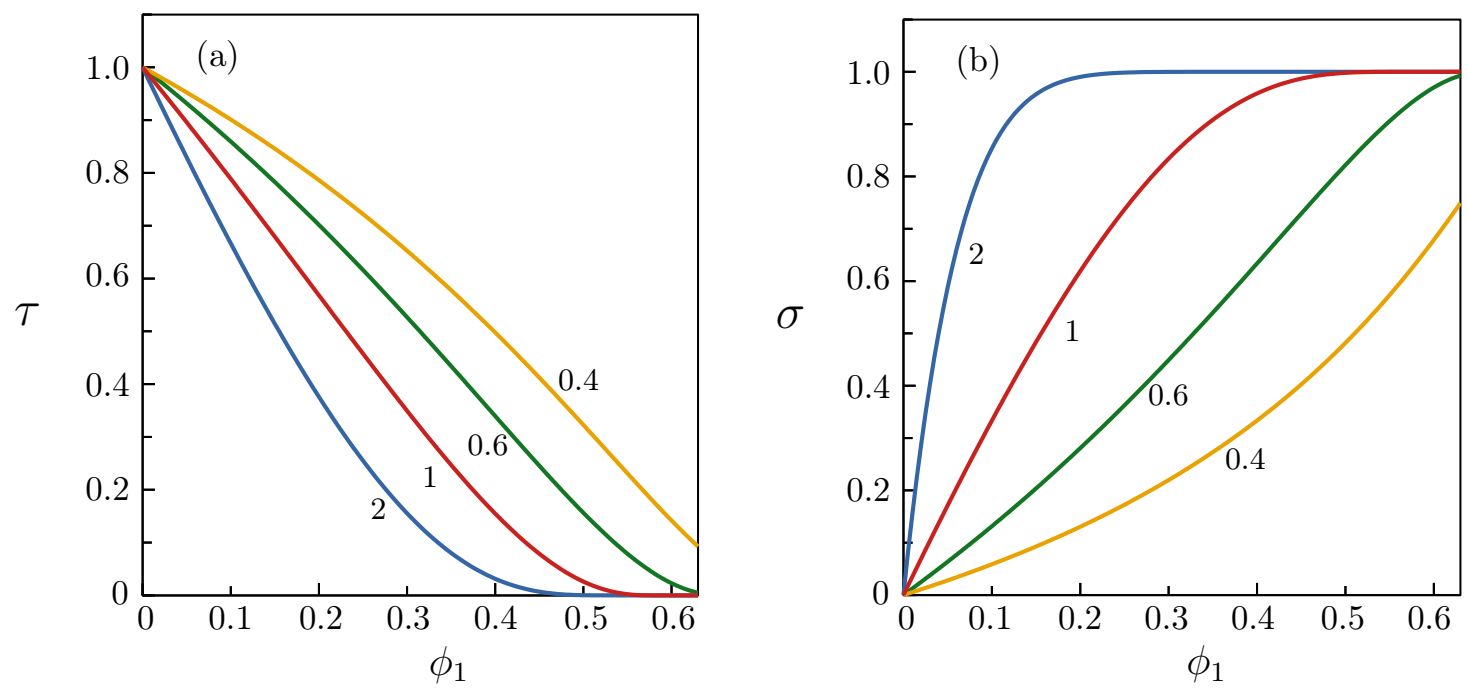

Figure 6: (a) Diffusive tortuosity factor $\tau$ and (b) colloidal reflection coefficient $\sigma$ as functions of $\phi_{1}$ for the case $\phi_{2}=0.001$ and four values of $\lambda$, corresponding to those in figure 5 .

Here $\lambda_{d}$ is the ratio of the tracer size $R_{2}$ to the locally averaged pore size within the suspension, $R_{d}=c_{d} R_{p}$, where $R_{p}=3 \phi_{1} /\left[R_{1}\left(1-\phi_{1}\right)\right]$ is the physical pore size [19]. The quantity $c_{d}$ is a scaling factor accounting for fluctuations in the pore size and $\phi_{g}=0.58$ is the volume fraction at the hard-sphere glass transition. The scaling factor ensures that $\tau \rightarrow \eta_{0} / \eta$ in the large tracer limit $R_{2} \rightarrow \infty$, where $\eta\left(\phi_{1}\right)$ is the dynamic viscosity of the host suspension. When $\lambda_{d} \geq 1$ the tracer particles are trapped in the pores and $\tau=0$ while $\sigma=1$ (figure 6).

This trapping effect is the physical cause of the filtration behaviour occurring in figures $3-5$. Setting $\lambda_{d}=1$ in $(23 a)$ gives $\phi_{1}^{c}=\left(1+3 \lambda / c_{d}\right)^{-1}$ as the critical colloid volume fraction at which particle trapping occurs [10]. As $\lambda$ decreases (smaller tracer particles), higher volume fractions are required to cause particle trapping [10] and for sufficiently small tracer particles trapping does not occur, as illustrated in figure 5 . The exponents $a_{\tau}$ and $a_{\sigma}$ in (22) are

$$
a_{\tau}=\frac{\tau_{1} c_{d}}{3 \lambda} \quad \text { and } \quad a_{\sigma}=\frac{\sigma_{1} c_{d}}{3 \lambda}
$$

where $\tau_{1}=2.5 \lambda /(.22+\lambda)$ and $\sigma_{1}=b_{12}-\left(1+3 \lambda+\lambda^{2}\right)+\lambda^{2} /\left(1+\lambda^{3}\right)$.

Figure 6 shows the diffusive tortuosity factor $\tau$ and reflection coefficient $\sigma$ as functions of $\phi_{1}$ for the case $\phi_{2}=0.001$ and four values of $\lambda$ corresponding to those in figure 5 . When $\lambda=2$ (blue curves), as $\phi_{1}$ increases $\tau \rightarrow 0$ while $\sigma \rightarrow 1$. When $\tau=0$ the tracer particles are unable to diffuse within the pore space, while when $\sigma=1$ the tracer particles are not able to advect within the pore space [10]. As $\lambda$ decreases, higher values of $\phi_{1}$ are required to block the tracer particles; this explains the curves in figure 5 , where the tracers are able to advect further into the membrane as $\lambda$ decreases. For $\lambda=0.4$ (yellow curves), the reflection coefficient $\sigma$ is less than one even at the close-packed limit; the tracer particles in figure 5 in this case (yellow curve) are therefore able to pass completely through the membrane and into the filtrate.

Finally, given $\alpha$ and $\sigma$, the cross-diffusion factor can be obtained as

$$
\ell=\frac{(\sigma+\alpha-1)}{\alpha} \text { and } \hat{\ell}=\frac{\phi_{2} \ell}{\lambda^{2} \phi_{1}} .
$$

With equations (19)-(25), the diffusion coefficients in (18) can be determined as functions of the volume fractions $\phi_{1}$ and $\phi_{2}$ and the particle size ratio $\lambda$. Figure 7 shows the $D_{i j}$ as functions of $\phi_{1}$ for the case $\lambda=2$ and $\phi_{2}=0.001$. The profiles are qualitatively similar to those obtained previously for the hard-sphere system $[10,18]$ and for salt diffusion in polymer solutions $[12,20]$. A significant aspect of these profiles is the fact that the tracer Fickian diffusivity $D_{22}$ approaches zero 

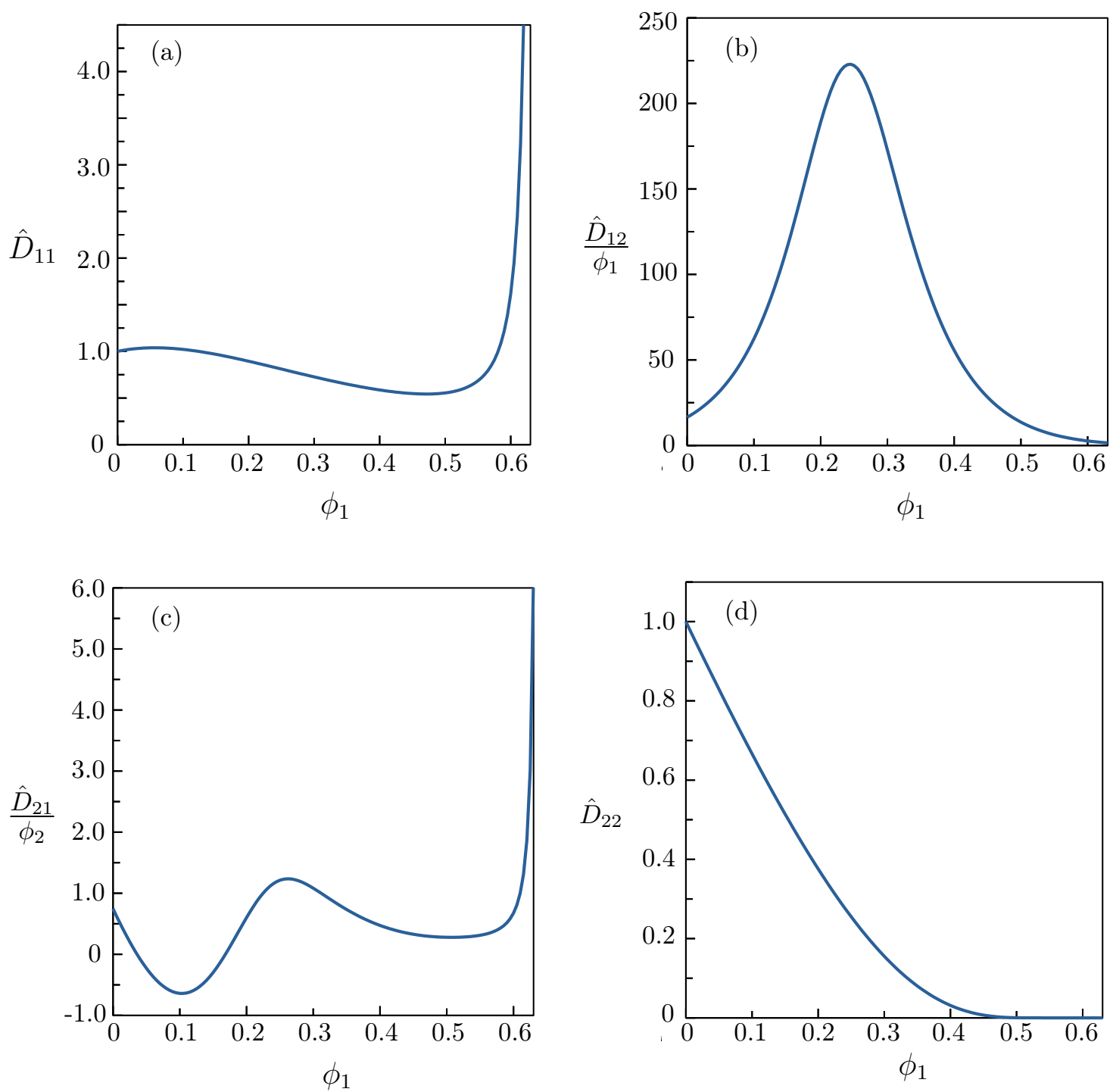

Figure 7: Cross-diffusion coefficients $\hat{D}_{i j}=D_{i j} / D_{i}^{0}$ as functions of volume fraction $\phi_{1}$ for the case $\lambda=2$ and $\phi_{2}=0.001$.

in the close-packed limit $\phi_{1} \rightarrow \phi_{p}$, while the tracer cross-diffusion coefficient $D_{21}$ remains positive. Therefore the tracer motion is determined by cross-diffusion in this limit. This effect enables the colloidal membrane to stop the advection of the tracer particles, as discussed further in Section 4 .

\section{References}

[1] R. W. Baker. Membrane Technology and Applications Third Edition. Wiley, New York, 2012.

[2] A. Katzir-Katchalsky and P. F. Curran. Nonequilibrium Thermodynamics in Biophysics. Harvard University Press, Boston, 1965.

[3] L. W. Henderson. Biophysics of ultrafiltration and hemofiltration. In Jacobs C., Kjellstrand C.M., Koch K.M., and Winchester J.F., editors, Replacement of Renal Function by Dialysis, pages 114-145. Springer, Dordrecht, 1996. 
[4] C. Y. Tang, T. H. Chang, and A. G. Fane. Colloidal interactions and fouling of nf and ro membranes: A review. Advances in Colloid and Interface Science, 164, 126-143, 2011.

[5] M. E. Ersahin, H. Ozgun, R. K. Dereli, I. Ozturk, K. Roest, and J. B.van Lier. A review on dynamic membrane filtration: Materials, applications and future perspectives. Bioresource Technology, 122, 196-206, 2012.

[6] Jingwei Wang, Andy Cahyadi, Bing Wu, Wenxi Pee, Anthony G. Fane, and Jia Wei Chew. The roles of particles in enhancing membrane filtration: A review. Journal of Membrane Science, 595, 117570, 2020.

[7] Aditya Anantharaman, Youngpil Chun, Tao Hua, Jia Wei Chew, and Rong Wang. Predeposited dynamic membrane filtration - A review. Water Research, 17, 115558, 2020.

[8] Jessa Marie J. Millanar-Marfa, Laura Borea, Fabiano Castrogiovanni, Shadi Wajih Hasan, Kwang-Ho Choo, Gregory V. Korshin, Mark Daniel G. de Luna, Florencio C. Ballesteros Jr., Vincenzo Belgiorno, and Vincenzo Naddeo. Self-forming dynamic membranes for wastewater treatment. Separation \& Purification Reviews, 0, 1-17, 2021.

[9] G. K. Batchelor. Diffusion in a dilute polydisperse system of interacting spheres. Journal of Fluid Mechanics, 131, 155-175, 1983.

[10] S. S. L. Peppin. Theory of tracer diffusion in concentrated hard-sphere suspensions. J. Fluid Mech., 870, 1105-1126, 2019.

[11] S. S. L. Peppin. Anomalous tracer diffusion in hard-sphere suspensions. engrXiv, https://engrxiv.org/nm95k, 2021.

[12] S. S. L. Peppin. Effective hard-sphere model of diffusion in aqueous polymer solutions. OSF Preprints, https://osf.io/9yuje, 2021.

[13] K. E. Davis and W. B. Russel. An asymptotic description of transient settling and ultrafiltration of colloidal dispersions. Physics of Fluids A: Fluid Dynamics, 1, 82-100, 1989.

[14] K. A. Landman and W. B. Russel. Filtration at large pressures for strongly flocculated suspensions. Physics of Fluids A: Fluid Dynamics, 5, 550-560, 1993.

[15] J. L. Anderson and J. A. Quinn. Restricted transport in small pores: A model for steric exclusion and hindered particle motion. Biophysical Journal, 14, 130-150, 1974.

[16] Gaurav Bhalla and William M. Deen. Effects of molecular shape on osmotic reflection coefficients. Journal of Membrane Science, 306, 116-124, 2007.

[17] G. K. Batchelor. Sedimentation in a dilute dispersion of spheres. Journal of Fluid Mechanics, 52, 245-268, 1972.

[18] W. B. Russel, D. A. Seville, and W. R. Schowalter. Colloidal Dispersions. Cambridge University Press, U.K., 1989.

[19] R. B. Bird, W. E. Stuart, and E. N. Lightfoot. Transport Phenomena Second Edition. Wiley, N.Y., 2002.

[20] A. Vergara, O. Annunziata, L. Paduano, D. G. Miller, J. G. Albright, and R. Sartorio. Multicomponent diffusion in crowded solutions. 2. Mutual diffusion in the ternary system tetra(ethylene glycol)-NaCl-water. The Journal of Physical Chemistry B, 108, 2764-2772, 2004. 